\title{
Comparative effects of two species of floricolous Metschnikowia yeasts on nectar
}

\author{
Azucena Canto $^{1 *}$, Carlos M. Herrera ${ }^{2}$, Isabel M. García ${ }^{2}$, Marina García ${ }^{2}$, Pilar Bazaga ${ }^{2}$, \\ ${ }^{1}$ Centro de Investigación Científica de Yucatán, A.C.C. 43 No. 130, Chuburná de Hidalgo, 97200 Mérida Yucatán, México; azucanto@cicy.mx \\ ${ }^{2}$ Estación Biológica de Doñana, Consejo Superior de Investigaciones Científicas, Avda. Americo Vespucio s/n, 41092 Sevilla, Spain; herrera@ebd.csic.es; \\ imagajim@ebd.csic.es; marina@ebd.csic.es; pbazaga@ebd.csic.es.
}

\begin{abstract}
Canto, A., Herrera, C.M., García, I.M., García, M., Bazaga, P. 2015. Comparative effects of two species of floricolous Metschnikowia yeasts on nectar. Anales Jard. Bot. Madrid 72(1): e019

Nectar yeast communities in southern Spain are dominated by two closely-related species, Metschnikowia reukaufii Pitt \& M.W. Mill. and M. gruessii Gim.-Jurado (Ascomycota, Saccharomycetales), although they tend to be distributed differentially across different host plants. We explore here the possibility that the two yeasts play different functional roles in floral nectar by differing in their impact on sugar concentration and composition of nectar. Experiments were undertaken under controlled conditions using bumblebees caught foraging on the flowers of two different host plants each of which is known to harbor predominantly one of the two yeasts. Bumblebees were used as sources of inocula to obtain two groups of samples from the nectar of Helleborus foetidus L. (Ranunculaceae): nectar samples inoculated with $M$. gruessii and samples inoculated with $M$. reukaufii. Metschnikowia gruessii was poorly represented in nectar samples, while $M$. reukaufii was by far the most common and had the highest cell density. Although the two yeasts caused relatively similar changes in nectar sugar composition, which involved increasing fructose and decreasing sucrose proportions, they marginally differed in their quantitative impact on total nectar sugar concentration. Results suggest that differential yeast occurrence across host plants may lead to yeast specialization and modify the outcomes of the plant-pollinator interface.
\end{abstract}

Key words: Bombus terrestris, fructose, Helleborus foetidus, nectar-living yeasts, Rosmarinus officinalis, sucrose

\section{INTRODUCTION}

Floral nectar is a sugar-dominated secretion that plants offer to floral visitors in exchange for pollination services (Simpson \& Neff, 1983). Nectar is a suitable growing medium for microorganisms that is often colonized by nectar-living yeasts frequently harbored in the pollinator's mouth parts. Vegetative yeast cells are transported to nectaries attached to the mouth parts of floral visitors. For example, European bumblebees harbor particular yeasts that are phylogenetically related and which avidly proliferate and chemically change the floral nectar of bumblebee-visited plants (BryschHerzberg, 2004; Canto \& al., 2008; Herrera \& al., 2008). In southern Spain, nectar yeast communities are dominated by Metschnikowia reukaufii and M. gruessii (Pozo \& al., 2011; Álvarez-Perez \& Herrera, 2013), which tend to be distributed differentially across host plant species. A survey conducted by Pozo \& al. (2011) showed that $M$. reukaufii was more common than $M$. gruessii in the floral nectar of 16 plant

\section{Resumen}

Canto, A., Herrera, C.M., García, I.M., García, M., Bazaga, P. 2015. Comparación de efectos de dos especies de levaduras florícolas Metschnikowia en el néctar. Anales Jard. Bot. Madrid 72(1): e019

Las comunidades de levaduras asociadas al néctar en el sur de España son dominadas por dos especies, Metschnikowia reukaufii Pitt \& M.W. Mill. y M. gruessii Gim.-Jurado (Ascomycota, Saccharomycetales). Ambas levaduras son especies muy cercanas entre sí, pero se distribuyen diferencialmente entre las especies de plantas cuyo néctar las hospeda. En este trabajo se explora la posibilidad de que las levaduras tengan funciones distintas impactando diferencialmente la concentración y composición del néctar. Para esto se realizaron experimentos bajo condiciones controladas usando individuos de abejorros que fueron capturados cuando visitaban flores que albergan predominantemente una de las dos posibles levaduras mencionadas. Los abejorros fueron utilizados como agentes inoculantes naturales en el néctar de Helleborus foetidus L. para obtener dos grupos de muestras, unas con $M$. reukaufii y otras con $M$. gruessii. Los resultados mostraron que M. gruessii estuvo pobremente representada en las muestras; en contraste, $M$. reukaufii fue la más frecuente y de mayor densidad en el néctar. Aun cuando ambas levaduras tuvieron un efecto similar en la composición proporcional de los azúcares en el néctar, aumento de la fructosa y decremento de la sacarosa, las dos especies difieren marginalmente en su impacto en la concentración total de azúcares. Los resultados sugieren que la ocurrencia diferencial de las especies de levaduras entre sus plantas hospederas, puede conducir a una modificación en las interacciones entre las plantas y sus visitantes florales y también a una especialización de levaduras por hospedero.

Palabras clave: Bombus terrestris, fructosa, Helleborus foetidus, levaduras nectarícolas, Rosmarinus officinalis, sacarosa

species while the reverse was true for 7 host species. Under controlled laboratory conditions, commercial Bombus terrestris L. workers are able to discriminate between nectars containing the two yeast species, being more attracted to nectars containing $M$. reukaufii than those containing $M$. gruessii (Herrera \& al., 2013). These two yeast species are distinct phenotypically, differing in cell and ascus morphology and physiology, e.g., assimilation of trehalose, osmotolerance range and maximum temperature for growth (Barnett \& al., 2000; Giménez-Jurado, 1992; Lachance 2011).

Together, these observations suggest that $M$. reukaufii and M. gruessii may play different functional roles in floral nectar composition, an aspect that has not previously been explored. As an initial approach, we explore here whether the two dominant nectar yeasts $M$. reukaufii and $M$. gruessii differ in their impact on floral nectar sugar concentration and composition under controlled conditions. To this end, experiments were undertaken using bumblebees caught foraging on the flowers of two simultaneously flowering host 
plants, each of which is known to harbor predominantly one of the two yeasts. Metschnikowia reukaufii is frequently found in the nectar of Helleborus foetidus, whereas M. gruessii is common in the nectar of Rosmarinus officinalis $\mathrm{L}$. (Pozo \& al., 2011). Using the natural vector for inoculate nectar made it possible to maintain the natural variation of yeast-cell quantity that bumble bees carry to nectaries. We specifically addressed whether the two yeasts differed in their effects on nectar sugars composition and total nectar sugar concentration, and in the population density reached in floral nectar.

\section{MATERIALS AND METHODS}

\section{Study site and nectar source}

This study was conducted during February-March 2008 in the Cazorla-Segura-Las Villas Natural Park in Jaén province, southeastern Spain, an area characterized by well-preserved pine-oak montane forests and woodlands. Natural nectar was obtained from nectaries of Helleborus foetidus (Ranunculaceae), a perennial, bumblebee-pollinated herb widely distributed in western and southwestern Europe (Weber \& Ebel, 1994). Nectar was collected from plants growing naturally on woodland slopes surrounding the Roblehondo field station, where the species flowers during January-March. When this study was undertaken, a large number of plants were in bloom in the area and thus available for nectar collection. Each flower generally contains between one and eight individual nectaries, derived from reduced and modified petals. These nectaries have a flattened horns morphology and are placed deeply hidden inside the greenish perianth, forming a ring between the stamens and sepals (Herrera \& al., 2001). Each nectary produces on average $2 \mu \mathrm{L}$ of nectar which has high sugar concentrations, with an overall value of $59.7 \pm 19.4 \mathrm{~g}$ $100 \mathrm{~mL}^{-1}$. Nectar is sucrose-dominated $(98.5 \%)$ with low percentages of glucose $(0.2 \%)$ and fructose $(1.3 \%$; Canto \& al., 2011).

\section{Sampling methods}

Nectar was obtained from ten spatially separated plants of $H$. foetidus that were covered with tulle fabric when their inflorescences were in bud to exclude pollinators. During flowering, two or three freshly opened flowers with nectaries full of nectar were collected from each plant, pooled in a container and kept refrigerated following the procedure of Canto \& al. (2008). From these, a random sample of flowers was used in the inoculation experiments, generally within a few hours after collection. Since the two yeast species are delivered to floral nectar by bumblebees introducing their proboscis into nectaries in search of nectar (Canto \& al., 2008), in order to obtain the desired yeast, individuals of Bombus terrestris were hand-netted while they were visiting one of two flower sources: flowers of $H$. foetidus to acquire cells of $M$. reukaufii or flowers of $R$. officinalis to obtain cells of $M$. gruessii. The two plant species grow in different environments and for this study, these two flower sources were approximately $10 \mathrm{~km}$ apart in a mountain range to avoid overlapping between the two bees populations. Trapped bees were used as source of inocula to obtain two groups of nectar samples with their respective control samples. Immediately after being captured, bees were individually placed in sterile containers and anaesthetized by cooling them at $4{ }^{\circ} \mathrm{C}$. The glossa of each individual bumblebee was carefully extended beyond the tip of the maxillary galeae and briefly introduced into a nectary of one of the previously selected flowers of $H$. foetidus containing its own naturally produced nectar. Another nectary in the same flower was marked and left untouched as the control reference for microbial growth. This method was repeated for 30 bumblebees and 30 individual flowers, in order to obtain 15 inoculated nectar samples with $M$. reukaufii and 15 with $M$. gruessii and 30 control samples. Inoculated and control samples were incubated at $25^{\circ} \mathrm{C}$ during $48 \mathrm{~h}$, after which the nectar contained in each nectary was divided into three subsamples: the first $(0.5 \mu \mathrm{L})$ was examined under a microscope at $\times 400$ and $\times 1000$ using a Neubauer chamber to verify microbial presence and estimate directly yeast cell density (cells $\mu \mathrm{L}^{-1}$ ) according to the methods by Herrera $\&$ al. (2009). The second subsample $(0.5 \mu \mathrm{L})$ was placed into a $1.5 \mathrm{~mL}$ sterile vial, diluted with HPLC-grade water to $1 \mathrm{~mL}$ of volume and kept frozen until the high-performance liquid chromatography (HPLC) analysis was performed to determine its sugar composition. Finally, the third subsample was used to verify the presence of yeasts and obtaining isolates for molecular species identification. The sample was streaked onto Yeast Malt agar plates $(1.0 \%$ glucose, $0.5 \%$ peptone, $0.3 \%$ malt extract, $0.3 \%$ yeast extract, $2.0 \%$ agar, $\mathrm{pH}=6.0)$ with $0.01 \%$ chloramphenicol, and incubated at $25^{\circ} \mathrm{C}$ for two weeks. $1-5$ single colonies were picked up from each culture agar plate and re-cultured for isolation, and then strains were identified by DNA sequencing (Herrera $\&$ al., 2010). For HPLC analyses each nectar-containing vial was treated according to the procedure described in Canto $\&$ al. (2011). Only sucrose, glucose, and fructose appeared regularly in nectar subsamples, and their proportions were estimated by integrating areas under chromatogram peaks. To calculate sugar concentration for single nectar subsamples calibration curves were constructed using linear regressions between sugar concentration of standard solutions of the three sugars and the integrated area in the corresponding chromatograms. Resulting concentrations were then summed and corrected according to initial sampling volume $(0.5 \mu \mathrm{L})$ to obtain the total sugar concentration $\left(\mathrm{g} 100 \mathrm{~mL}^{-1}\right)$ per subsample.

\section{Statistical analyses}

To test for differences in microbial cell density (log transformed) and total sugar concentration (arcsine transformed) between inoculated and control nectar samples, and between yeast species, a linear mixed model and contrast tests were performed with the MIXED procedure of the SAS statistical program (SAS Institute, Cary, North Carolina, USA). Because the experiment was conducted using pairs of nectaries within flowers (inoculated and control nectary in the same flower), the flower level was included in the model as a random effect. To test the respective differences in the composition of nectar sugar and the correlative association among sugar proportions, a MANOVA analysis was performed together with a Pearson's correlation coefficient calculation. 


\section{RESULTS}

Examination under a microscope showed that $M$. reukaufii developed in 14 of the 15 inoculated nectar samples. In 12 samples it was the only yeast present, while in the remaining two samples it developed along with $M$. gruessii and Hanseniaspora sp (one instance each). There was no microbial development at all in one sample. In the two samples with two yeast species, the numerically dominant yeast was $M$. reukaufii, as confirmed by their predominance in several isolates identified from each sample.

Metschnikowia gruessii was the only yeast developed in 5 of the 15 inoculated samples, Candida bombi developed in one sample, and no co-occurrence of yeast species was detected. No bacteria were microscopically observed in nectar samples, but PCR amplification failure of fungal primers on DNA from one of the colonies assayed could perhaps reflect that they were bacteria rather than yeasts. Cell density was higher in the samples containing $M$. reukaufii than in those with $M$. gruessii (mean \pm SD: $52148 \pm 48728$ cells $\mu \mathrm{L}^{-1}$ versus $7129 \pm 2772$ cells $\mu \mathrm{L}^{-1}$; $\mathrm{t}=11.29, \mathrm{df}=21, p<0.0001$ ).

Overall, total sugar concentration differed significantly between inoculated and control nectar samples $\left(\mathrm{F}_{1,21}=54\right.$, $p<0.0001)$. Average sugar concentration in the inoculated samples was less than half that of the control samples

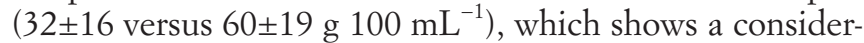
able reduction in sugar content induced by yeasts over a relatively short experimental period. No differences in sugar concentration were observed between samples containing different yeast species $\left(F_{1,21}=0.15, p=0.70\right)$. However, the average sugar concentration of samples inoculated with M. reukaufii $\left(29 \pm 16 \mathrm{~g} 100 \mathrm{~mL}^{-1}\right)$ was slightly lower than of samples with $M$. gruessii $\left(36 \pm 16 \mathrm{~g} 100 \mathrm{~mL}^{-1}\right)$, which suggest a more extensive sugar depletion by the former species.

Sugar composition differed significantly between control and inoculated samples (Wilks' $\lambda=0.09, \mathrm{~F}_{1,42}=414.04$, $p<0.0001)$. While control samples were sucrose-dominated with similar proportions of glucose and fructose, inoculated samples, irrespective of the yeast species, were fructosedominated with dissimilar proportions of glucose and sucrose (Fig. 1). Although the difference in sugar composition between

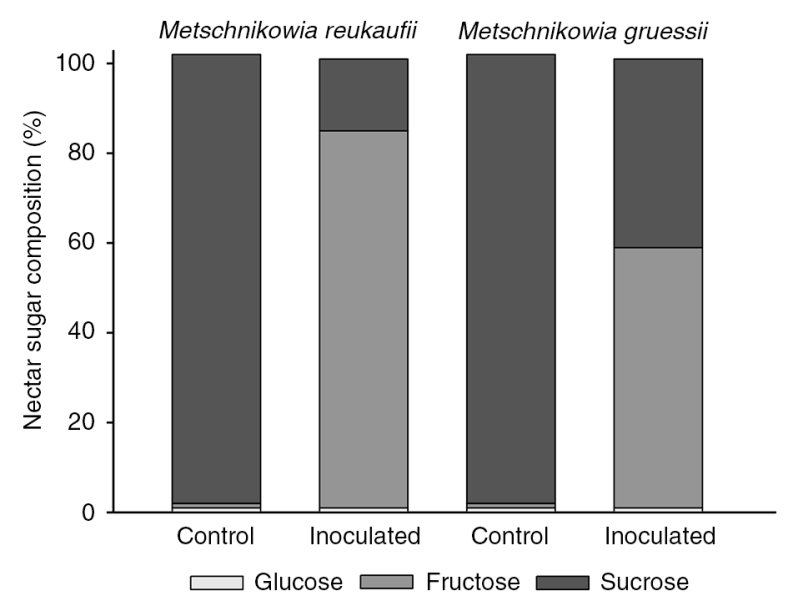

Fig. 1. Differences in sugar composition in nectar samples of Helleborus foetidus as a result of the yeast-inoculation experiment using the two nectar-dwelling yeasts Metschnikowia reukaufii and M. gruessii. Average percentage of each sugar in nectar is shown for inoculated and control samples. nectar samples with M. gruessii and M. reukaufii was not significant (Wilks' $\lambda=0.99, \mathrm{~F}_{1,42}=0.06, p=0.81$ ), nectar samples with $M$. reukaufii tended to have proportionally more fructose and lower sucrose than those with M. gruessii (Fig. 1). After inoculation with yeasts, proportions of those sugars were inversely related: as fructose increased, sucrose decreased by the same magnitude $(\mathrm{r}=-1, p<0.0001, \mathrm{~N}=46)$, but glucose proportion in nectar remained unchanged $(\mathrm{r}=0, p>0.05$, $\mathrm{N}=46$; Fig. 1).

\section{DISCUSSION}

Although inadvertent inoculation of bacteria along with predominant yeasts cannot be entirely ruled out, circumstantial evidence tends to suggest that, if at all present in our inoculated nectar samples, the density of bacteria should have been negligible. Firstly, bacteria were never detected in our microscopical $(\times 400$ and $\times 1000$ magnification $)$ observations of both inoculated and control samples. And secondly, prior culture-based assessment of yeast and bacteria abundance in $H$. foetidus and $R$. officinalis has shown that, even when present, bacteria are generally an unimportant component of the microbial communities in the nectar of these species (Álvarez-Pérez \& Herrera, 2013). Furthermore, the presence of yeasts other than $M$. reukaufii and M. gruessii in inoculated samples was too occasional to have any important effect on our results. For instance, C. bombi had not effect on nectar in the single sample where it was the only yeast, and changes in sugar composition observed in the two samples where $M$. reukaufii developed together with either M. gruessii or Hanseniaspora sp. were similar to changes in samples where $M$. reukaufii was the only yeast present.

There is a possibility that the handling technique used in flowers (see Sampling methods), could have triggered chemical changes in nectar before the inoculation procedure in such a way, that the actual effects of yeast on nectar could be hiding. This inoculation method was previously used by Canto \& al. (2008) and Canto \& al. (2011), having qualitatively similar results when flowers were inoculated with yeasts, no matter whether the flowers were left in plants or handled in lab conditions, or yeasts were inoculated to artificial sugar solutions mimicking floral nectar of $H$. foetidus. Moreover, flowers used here were cut from plants when nectaries were already full of nectar and processed soon after collection, avoiding a possible chemical changes. We are therefore confident that the results reported in this study are not influenced by the presence of other microbes in experimental nectar samples, or by chemical changes triggered by the experimental manipulation of flowers, mainly reflecting the impact of $M$. reukaufii and $M$. gruessii on nectar.

Our results suggest that, despite their phylogenetic relatedness, $M$. reukaufii and $M$. gruessii are not functionally equivalent in terms of their effects on floral nectar impact and in the cell densities reached when tested on the same nectar. Although the two species quite rarely occur together in nectar of $H$. foetidus, they do co-occur in nectars of other plant species across the study area (Pozo \& al., 2011). According to Lachance (2011), the physiological distinction between the two yeast species is not clear-cut based on growth performance in artificial culture media. For example, growth on trehalose is absent in M. gruessii, but it ranges from absent to slow in M. reukaufii. Assimilation of 
methyl- $\alpha$-D-glucoside is absent in $M$. gruessii whereas the response for $M$. reukaufii is weak to slow. Growth on 2-keto-D-gluconate, D-glucosamine, and hexadecane, as well as the hydrolysis of gelatin and casein hydrolysis and growth in the presence of $50 \%$ glucose, varied from weak to strong in both species. From this relative physiological similarity, therefore, the two yeasts would be expected to have roughly comparable effects on nectar traits. Our results indicate, however, that they differed in their ability to proliferate in $H$. foetidus nectar. This suggests that the two species of nectar-living yeasts differed in their growth rate in nectar. It is important to note, however, that our experimental design did not allow controlling for initial differences in cell densities in inoculated nectar samples, a factor that could perhaps have also contributed to observed interspecific differences in final cell densities.

Despite their overall similarity in physiological attributes noted above, the two yeast species differ in cell morphology, maximum temperature for growth and the ability to grow at extreme osmotic pressures (Giménez-Jurado, 1992; Barnett \& al., 2000). In this study $M$. reukaufii grew in all inoculated nectar samples and presented the highest cell density; in contrast, M. gruessii grew very little and only in a few samples. Both yeasts grew in culture media at $50 \%$ w/w glucose (Barnett \& al., 2000; Pozo \& al., 2012), but at $60 \%$ glucose $M$. gruessii is inhibited, whereas $M$. reukaufii is capable of growing rapidly (Giménez-Jurado, 1992). The nectar samples of $H$. foetidus used in this study had an average sugar concentration of $60 \%$. Consequently, M. gruessii cells had to cope after inoculation with an extreme osmotic pressure, which would explain its poor growth in the nectar samples. The floral nectar of $R$. officinalis was nearly as sucrose-dominated as that of $H$. foetidus ( $75 \%$ sucrose; C. M. Herrera, unpublished), but its average sugar concentration, measured in 13 flowers from plants growing at the study site, was considerably lower $(34 \% \mathrm{w} / \mathrm{w})$. Therefore, the strains of $M$. gruessii studied here originated from environments where osmotic pressure was considerably less adverse than those faced later in the experiment using nectar of $H$. foetidus. On the other hand, the two yeasts induced similar qualitative changes in terms of nectar sugar composition. Sugar proportions changed following similar patterns in all inoculated nectar samples irrespective of yeast species. Therefore, the marginal trend for $M$. reukafii increasing the relative fructose content to a greater extent than $M$. gruessii, needs to be corroborated by including nectar samples of $R$. officinalis. Because the low volume of nectar produced by flowers of $R$. officinalis we failed obtaining the three nectar aliquots required to conduct chemical analysis, cell counting method and microbial culturing. Notwithstanding this experimental limitation, the results are compatible with the notion that floral niche differentiation between these two yeasts species is made possible by a differential ability to exploit nectar because of differences in osmotolerance.

Metschnikowia reukaufii has important levels of phenotypic plasticity that allow it to colonize a broad range of nectar types, including some representing extreme environments (Herrera \& al., 2011; 2012). For example, H. foetidus nectar imposes harsh conditions for microbial growth because of the high sugar concentration and presence of antimicrobial substances (Herrera \& al., 2006; Canto \& al., 2011). Under such conditions $M$. reukaufii usually reaches considerably higher cell densities than M. gruessii. Differential responses to toxic substances in nectar may also contribute to growth differences between yeast species, as they may act as barriers against yeast colonization (Escalante-Pérez \& Heil, 2013). The presence of protoanemonin, an antifungal lactone (Canto A \& Herrera CM unpublished results), in $H$. foetidus nectar might have influenced $M$. reukaufii and $M$. gruessii differentially, but this hypothesis remains to be explored. Irrespective of the underlying mechanisms, however, differences between the two yeast species in their development and occurrence in nectar documented in this paper can account for the different responses of pollinators to their presence (Herrera \& al., 2013) and ultimately lead to differential effects on pollination.

\section{ACKNOWLEDGEMENTS}

The authors thank Monica Medrano for her comments on the sampling design and Pedro A. Tíscar and the Centro de Capacitación y Experimentación Forestal de Vadillo-Castril for laboratory facilities. Permission to carry out the field work was granted by the Consejería de Medio Ambiente, Junta de Andalucía. This work was funded by grants P06-RNM-01627 (Consejería de Innovación, Ciencia y Empresa, Junta de Andalucía), CGL2006-01355, and EXPLORA CGL2007-28866-E/BOS (Ministerio de Educación y Ciencia, Gobierno de España).

\section{REFERENCES}

Álvarez-Pérez, S. \& Herrera, C.M. 2013. Composition, richness and nonrandom assembly of culturable bacterial-microfungal communities in floral nectar of Mediterranean plants. FEMS Microbiology Ecology 83: 685-699. http://dx.doi.org/10.1111/1574-6941.12027

Barnett, J.A., Payne, R.W. \& Yarrow, D. 2000. Yeasts: Characteristics and identification, third Ed. Cambridge University Press, Cambridge.

Brysch-Herzberg, M. 2004. Ecology of yeasts in plant-bumblebee mutualism in Central Europe. FEMS Microbiology Ecology 50: 87-100. http:// dx.doi.org/10.1016/j.femsec.2004.06.003

Canto, A., Herrera, C.M., García, I.M., Pérez, R. \& Vaz, M. 2011. Intraplant variation in nectar traits in Helleborus foetidus (Ranunculaceae) as related to floral phase, environmental conditions and pollinator exposure. Flora 206: 668-675. http://dx.doi.org/10.1016/j.flora.2011.02.003

Canto, A., Herrera, C.M., Medrano, M., Pérez, R. \& García, I.M. 2008. Pollinator foraging modifies nectar sugar composition in Helleborus foetidus (Ranunculaceae): An experimental test. American Journal of Botany 95: 315-320. http://dx.doi.org/10.3732/ajb.95.3.315

Escalante-Pérez, M. \& Heil, M. 2013. The production and protection of nectars. In: U. Lüttge \& al. (eds.), Progress in Botany 74: 239-261. Springer-Verlag, Berlin Heidelberg. http://dx.doi.org/10.1007/978-3-642-30967-0_9

Giménez-Jurado, G. 1992. Metschnikowia gruessii sp. nov., the teleomorph of Nectaromyces reukaufii but not of Candida reukaufii. Systematic and Applied Microbiology 15: 432-438. http://dx.doi.org/10.1016/S07232020(11)80218-3

Herrera, C.M., Canto, A., Pozo, M.I. \& Bazaga, P. 2010. Inhospitable sweetness: nectar filtering of pollinator-borne inocula leads to impoverished, phylogenetically clustered yeast communities. Proceedings of the Royal Society B: Biological Sciences 277: 747-754. http://dx.doi.org/10.1098/ rspb.2009.1485

Herrera, C.M., de Vega, C., Canto, A. \& Pozo, M.I. 2009. Yeasts in floral nectar: a quantitative survey. Annals of Botany 103: 1415-1423. http:// dx.doi.org/10.1093/aob/mcp026

Herrera, C.M., García, I.M. \& Pérez, R. 2008. Invisible floral larcenies: microbial communities degrade floral nectar of bumble bee-pollinated plants. Ecology 89: 2369-2376. http://dx.doi.org/10.1890/08-0241.1

Herrera, C.M., Pérez, R. \& Alonso, C. 2006. Extreme intraplant variation in nectar sugar composition in an insect-pollinated perennial herb. American Journal of Botany 93: 575-581. http://dx.doi.org/10.3732/ ajb.93.4.575

Herrera, C.M., Pozo, M.I. \& Bazaga, P. 2011. Clonality, genetic diversity and support for the diversifying selection hypothesis in natural populations of a flower-living yeast. Molecular Ecology 20: 4395-4407. http:// dx.doi.org/10.1111/j.1365-294X.2011.05217.x

Herrera, C.M., Pozo, M.I. \& Bazaga, P. 2012. Jack of all nectars, master of most: DNA methylation and the epigenetic basis of niche width in 
a flower-living yeast. Molecular Ecology 21: 2602-2616. http://dx.doi. org/10.1111/j.1365-294X.2011.05402.x

Herrera, C.M., Pozo, M.I. \& Medrano, M. 2013. Yeasts in nectar of an early-blooming herb: sought by bumble bees, detrimental to plant fecundity. Ecology 94: 273-279. http://dx.doi.org/10.1890/12-0595.1

Herrera, C.M., Sánchez-Lafuente, A.M., Medrano, M., Guitián, J., Cerdá, X. \& Rey, P. 2001. Geographical variation in autonomous selfpollination levels unrelated to pollinator service in Helleborus foetidus (Ranunculaceae). American Journal of Botany 88: 1025-1032. http:// dx.doi.org/10.2307/2657084

Lachance, M.A. 2011. Metschnikowia Kamienski (1899). In: Kurtzman, C.P. \& al. (eds.), The yeast, a taxonomic study 1: 575-620. Elsevier, London.

Pozo, M.I., Herrera, C.M. \& Bazaga, P. 2011. Species richness of yeast communities in floral nectar of southern Spanish plants. Microbial Ecology 61: 82-91. http://dx.doi.org/10.1007/s00248-010-9682-x
Pozo, M.I., Lachance, M.A. \& Herrera, C.M. 2012. Nectar yeasts of two southern Spanish plants: the roles of immigration and physiological traits in community assembly. FEMS Microbiology Ecology 80: 281-293. http://dx.doi.org/10.1111/j.1574-6941.2011.01286.x

Simpson, B.B. \& Neff, J.L. 1983. Evolution and diversity of floral rewards. In: Jones, C.E. \& Little, R.J. (eds.), Handbook of experimental pollination biology 142-159. Van Nostrand Reinhold, New York.

Weber, K. \& Ebel, F. 1994. Zur lebensgeschichte der gattung Helleborus L. (Ranunculaceae). Flora 189: 97-130.

Associate Editor: Javier Diéguez Received: 4-XI-2014

Accepted: 9-I-2015 